\title{
NGHIÊN CỨU GIẢI PHÁP NÂNG CAO HIỆU QUẢ CÔNG TÁC CHUYỂN ĐỘ CAO QUA GIẾNG ĐÚNG XUỐNG HÂM KHI THI CÔNG CÁC CÔNG TRÌNH ĐƯờNG HẦM CÓ Độ SÂU LỚN
}

\author{
DIÊM CÔNG HUY \\ Viện Khoa học Công nghệ Xây dựng - Bộ Xây dựng
}

\section{Tóm tắt:}

Nội dung của bài báo trình bày một số kết quả nghiên cứu về đặc điểm và giải pháp kỹ thuật của công tác chuyển độ cao qua giếng đứng xuống hầm, một số kết quả đo thực nghiệm, từ đó lựa chọn phương pháp chuyển độ cao qua giếng đứng xuống hầm phù hợp nhất nhằm nâng cao hiệu quả công tác định hướng hầm khi thi công các công trình đường hầm có độ sâu lớn.

\section{1. Đặt vấn đề}

Khi thi công các công trình đường hầm, độ chính xác của công trình phụ thuộc rất nhiều vào công tác định hướng hầm. Nếu công tác định hướng đào hầm làm không tốt thì kết quả thông hầm sẽ không đạt yêu cầu hạn sai làm ảnh hưởng đến chất lượng, tiến độ thi công công trình. Do vậy vấn đề định hướng đường hầm là rất quan trọng trong công tác thi công đường hầm có độ sâu lớn.

Công tác định hướng hầm phải dựa vào cơ sở trắc địa thi công hầm bao gồm: lưới khống chế mặt đất, lưới khống chế trong hầm và công tác chuyền tọa độ và độ cao xuống hầm. Để đảm bảo cho công tác thông hầm đạt hiệu quả cao nhất và nằm trong giới hạn cho phép thì cần giải quyết được vấn đề nâng cao độ chính xác của cơ sở trắc địa trong thi công hầm. Do đặc điểm của công tác thi công hầm nên vấn đề đo đạc chuyền độ cao qua giếng đứng xuống hầm để thành lập lưới khống chế độ cao thi công trong hầm có nhiều phương pháp khác nhau. Do đó cần phải nghiên cứu lựa chọn phương pháp chuyển độ cao qua giếng đứng xuống hầm phù hợp nhất nhằm đảm bảo độ chính xác định hướng hầm và đáp ứng được các điều kiện thi công hầm trong thực tế phù hợp với tiến độ thi công công trình.

\section{Cơ sở lý thuyết và phương pháp thực hiện.}

\subsection{Cơ sở lý thuyết}

Nguyên lý của việc truyền độ cao từ trên mặt đất xuống dưới hầm là "Tạo ra hai mặt phẳng song song với nhau, một mặt phẳng ở trên mặt đất và một mặt phẳng ở dưới hầm". Nếu xác định được khoảng cách giữa hai mặt phẳng này thì sẽ truyền được độ cao từ trên mặt đất xuống dưới hầm.

\subsection{Phương pháp thực hiện}

Sơ đồ chuyền độ cao bằng máy toàn đạc điện tử qua giếng đứng xuống hầm được đưa ra (hình 1). Tại một vị trí trên mặt đất, cách giếng đứng một khoảng cách từ $10 \mathrm{~m}$ $\div 15$ m đặt một máy Toàn đạc điện tử $D$. Trên miệng giếng đặt một gương phẳng $\mathrm{P}$ có thể quay quanh một trục cố định tạo thành một góc tới $45^{\circ}$ so với phương nằm ngang là đường chuyền của tia sáng. (Xem hình 1)

Nhờ gương phẳng phụ $\mathrm{P}$ này mà tia sáng từ máy Toàn đạc điện tử đến gương

\footnotetext{
Ngày nhận bài: 03/11/2016, ngày chuyển phản biện: 07/11/2016, ngày chấp nhận phản biện: 21/11/2016, ngày chấp nhận đăng: 28/11/2016
} 
phẳng $\mathrm{P}$ được thay đổi hướng đi $90^{\circ}$ và hướng đến gương phản xạ $O$ đặt ở dưới hầm. Tia phản xạ trở lại gương $\mathrm{P}$ và bộ phận thu nhận ánh sáng của máy Toàn đạc điện tử [1].

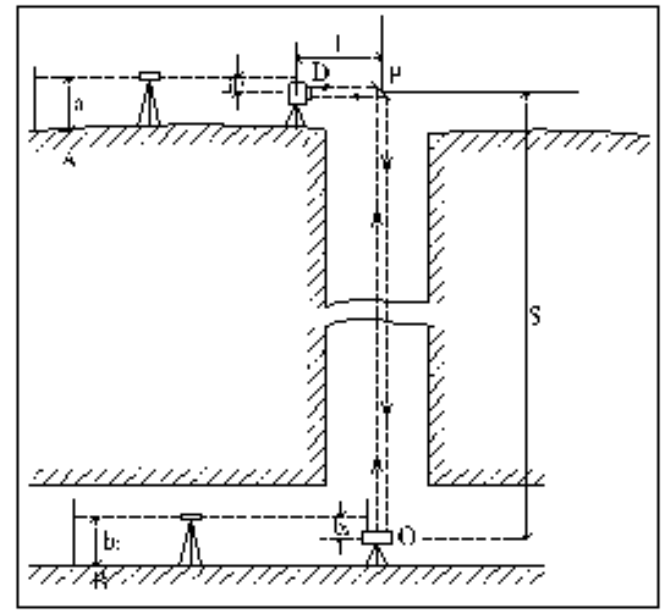

Hình 1: So đồ chuyền độ cao bằng máy toàn đạc điện tử qua giếng đứng xuống hầm

Trình tự đo đạc để chuyền độ cao từ mặt đất xuống hầm triển khai như sau:

- Từ điểm $A$ là điểm khống chế độ cao trên mặt đất có độ cao là $\mathrm{H}_{\mathrm{A}}$ dùng một máy thủy chuẩn để xác định độ cao của tâm phát sóng trên máy toàn đạc điện tử bằng cách đọc số trên mia dựng tại điểm $\mathrm{A}$ (số đọc $\mathrm{a}_{1}$ ) và số đọc của thước $\mathrm{mm}$ đặt tại tâm phát sóng của máy toàn đạc điện tử $\left(\mathrm{a}_{2}\right)$. Để gương phẳng $\mathrm{P}$ ở vị trí vuông góc với phương phát sóng và đo khoảng cách $\mathrm{I}$. Quay gương $P$ sang vị trí với tia sóng một góc $45^{\circ}$ để tia sóng xuống hầm và phản xạ quay trở lại máy toàn đạc điện tử, đo được khoảng cách $L$.

- Trong hầm tiến hành chuyền độ cao từ gương $O$ đến điểm $B$ là điểm khống chế độ cao trong hầm bằng cách sử dụng máy thủy chuẩn đọc số trên mia tại điểm $B$ (số đọc $b_{1}$ ) và đọc số trên thước đo tại gương phản xạ $\mathrm{O}$ (số đọc $\mathrm{b}_{2}$ ). Khi đó độ cao của điểm $\mathrm{B}$ trong hầm được tính theo công thức:

$$
\mathrm{H}_{\mathrm{B}}=\mathrm{H}_{\mathrm{A}}+\left(\mathrm{a}_{1}-\mathrm{a}_{2}\right)-(\mathrm{L}-\mathrm{I})-\left(\mathrm{b}_{1}-\mathrm{b}_{2}\right)
$$

Trong đó:

$\mathrm{H}_{\mathrm{A}}$ là độ cao của điểm $\mathrm{A}$ trên mặt đất;

$a_{1}, a_{2}$ là đọc số trên các mia trên mặt đất;

I là khoảng cách từ gương phẳng phụ đến máy đo khoảng cách;

L là khoảng cách đo được bằng máy đo dài từ máy đến gương $\mathrm{O}$ trong hầm;

$b_{1}, b_{2}$ là số đọc trên các mia trong hầm.

Công thức (1) có thể viết như sau:

$$
\mathrm{H}_{\mathrm{B}}=\mathrm{H}_{\mathrm{A}}+\mathrm{h}_{1}-\mathrm{h}_{2}-\mathrm{h}_{3}
$$

Trong đó:

$$
\begin{aligned}
& \mathrm{h}_{1}=\mathrm{a}_{1}-\mathrm{a}_{2} \\
& \mathrm{~h}_{2}=\mathrm{L}-\mathrm{I} \\
& \mathrm{h}_{3}=\mathrm{b}_{1}-\mathrm{b}_{2}
\end{aligned}
$$

- Đánh giá độ chính xác của phương pháp

Từ công thức (2) ta có:

$$
\mathrm{m}_{\mathrm{HB}}^{2}=\mathrm{m}_{\mathrm{HA}}^{2}+\mathrm{m}_{\mathrm{h} 1}^{2}+\mathrm{m}_{\mathrm{h} 2}^{2}+\mathrm{m}_{\mathrm{h} 3}^{2}
$$

Trong đó:

$\mathrm{m}_{\mathrm{HA}}$ là sai số liệu gốc của điểm độ cao trên mặt đất (Bỏ qua sai số liệu gốc, ta có $\mathrm{m}_{\mathrm{HA}}=0$ )

$\mathrm{m}_{\mathrm{h} 1}$ là sai số đo thủy chuẩn trên mặt đất $\mathrm{m}_{\mathrm{h} 2}$ là sai số đo cạnh bằng máy $\mathrm{T} Đ T$ $\mathrm{m}_{\mathrm{h} 3}$ là sai số đo thủy chuẩn ở dưới hầm.

Ta có: $\mathrm{m}_{\mathrm{h} 2}^{2}=\mathrm{m}_{\mathrm{L}}^{2}+\mathrm{m}^{2}$,

Theo nguyên tắc ảnh hưởng bằng nhau:

$\mathrm{m}_{\mathrm{L}}=\mathrm{m}_{1}$ vậy $\mathrm{m}_{\mathrm{h} 2}^{2}=2 \mathrm{~m}_{\mathrm{L}}^{2}=2 \mathrm{~m}_{\mathrm{S}}^{2}$

$m_{h 1}=m_{h 2}=2 m_{h}^{2}$ 
Thay vào (3) ta có: $\mathrm{m}_{\mathrm{HB}}^{2}=2 \mathrm{~m}_{\mathrm{h}}^{2}+2 \mathrm{~m}^{2} \mathrm{~S}$

$$
\Rightarrow \mathrm{m}_{\mathrm{HB}}=\mathrm{m}_{\mathrm{h}} \sqrt{2}+\mathrm{m}_{\mathrm{S}} \sqrt{2}
$$

Vậy độ chính xác của phương pháp này phụ thuộc vào độ chính xác đo thủy chuẩn ở trên mặt đất và dưới hầm $\left(\mathrm{m}_{\mathrm{h}}\right)$ và độ chính xác của máy TĐĐT dùng để đo $\left(\mathrm{m}_{\mathrm{S}}\right)$. Kết quả thực nghiệm cho thấy nếu dùng máy TĐĐT TCR405 có độ chính xác $\mathrm{m}_{\mathrm{S}}=2 \mathrm{~mm}$ $+2 \mathrm{ppm}$ để đo thì sai số trung phương chuyển độ cao đạt độ chính xác $\mathrm{m}_{\mathrm{h} 2}= \pm$ $1.3 \mathrm{~mm}$ [4].

Phương pháp chuyền độ cao xuống hầm bằng máy toàn đạc điện tử có nhiều ưu điểm vượt trội so với các phương pháp chuyền độ cao truyền thống, nhưng cần phải tiến hành đo thực nghiệm để khẳng định về độ chính xác và khả năng ứng dụng của phương pháp.

\section{3. Đo đạc và tính toán thực nghiệm}

Quá trình đo đạc thực nghiệm là để minh chứng cho khả năng ứng dụng của phương pháp dùng máy TĐĐT chuyền độ cao qua giếng đứng xuống hầm và độ chính xác đạt được. Công tác thực nghiệm được tiến hành với máy toàn đạc điện tử Leica TS 06 Plus có độ chính xác đo cạnh $\mathrm{m}_{\mathrm{s}}=1.5 \mathrm{~mm}+$ $2.0 \mathrm{ppm}$ và độ chính xác đo góc $\mathrm{m}_{\beta}=2$ ". Tiến hành đo với phương án theo sơ đồ đã thiết kế như hình 4 tại hố thang máy từ tầng
29 xuống tầng 01 (cốt 00 ), Block $\mathrm{HH} 4$ tại công trình 136 Hồ Tùng Mậu, Hà Nội, cụ thể tiến hành như sau:

- Đầu tiên đặt máy TĐĐT và gương phẳng $\mathrm{P}$ tại tầng 29 , Block $\mathrm{HH} 4$, xoay gương phẳng $\mathrm{P}$ vuông góc với phương truyền sóng của máy $T \boxminus \boxminus T$, để ống kính máy ở vị trí $90^{\circ}$, tiến hành bật tia Laser, đánh dấu được điểm $M$ trên gương phụ $P$ và đo khoảng cách từ máy TĐĐT tới $\mathrm{P}$ bằng chế độ đo không gương ta được khoảng cách I (bảng 1). Vì gương phẳng $\mathrm{P}$ khi xoay $45^{\circ}$ xuống tầng 1 yêu cầu độ chính xác rất cao nên chúng tôi dùng dây dọi để dọi từ điểm $M$ đã được đánh dấu trên gương phụ $P$ xuống tầng 1 , xác định được điểm $\mathrm{N}$ dưới tầng 1. Đặt gương phản xạ $\mathrm{O}$ trùng với điểm $N$ sau đó xoay gương phụ $P$ nghiêng úp xuống đến khi thấy tia laser ở gương phản xạ $\mathrm{O}$ dưới tầng 1 thì dừng lại và đo được khoảng cách $L=I+S$ (bảng 1 ) bằng chế độ đo có gương của máy TĐĐT. Dựa vào kết quả trên, ta tính được khoảng cách từ gương phụ $P$ đến gương phản xạ $O$ là $S$ (Xem bảng 1).

- Dùng máy thuỷ chuẩn đo chênh cao từ mốc $A$ đến tâm phát sóng máy TĐĐT được chênh cao là $+1.372 m$ và đo chênh cao giữa tâm gương phản xạ $\mathrm{O}$ đến mốc độ cao B là $-0.084 m$ theo sơ đồ đo ở hình 1 .

\section{Bảng 1: Kết quả đo khoảng cách bằng từ máy $T \boxminus \boxminus T$ đến gương phụ $P$ và gương phản xạ $O$}

\begin{tabular}{|l|c|c|c|}
\hline $\begin{array}{c}\text { Khoảng cách đo bằng máy } \\
\text { TĐĐT Leicar TS 06 Plus }\end{array}$ & $\begin{array}{c}\text { Đo khoảng cách từ máy } \\
\text { TĐĐT tới gương phẳng } \\
\text { phụ } \mathrm{P}\end{array}$ & $\begin{array}{c}\text { Đo khoảng cách từ máy } \\
\text { TĐĐT tới gương phản xạ } \\
\mathrm{O}\end{array}$ & $\begin{array}{c}\text { Khoảng cách từ gương } \\
\text { phẳng phụ } \mathrm{P} \text { đến gương } \\
\text { phản xạ O }\end{array}$ \\
\hline & $\mathrm{I}(\mathrm{m})$ & $\mathrm{L}(\mathrm{m})$ & $\mathrm{S}=\mathrm{L}-\mathrm{I}(\mathrm{m})$ \\
\hline lần 1 & 4.192 & 100.262 & \\
\hline lần 2 & 4.192 & 100.262 & \\
\hline lần 3 & 4.192 & 100.262 & $\mathbf{9 6 . 0 7 0}$ \\
\hline Trung bình & $\mathbf{4 . 1 9 2}$ & $\mathbf{1 0 0 . 2 6 2}$ & \\
\hline
\end{tabular}



thức.

- Độ cao của điểm B được tính theo công

$H_{B}=H_{A}+\left(a_{1}-a_{2}\right)-(L-l)-\left(b_{1}-b_{2}\right)$

Thay số vào ta có:

$$
\begin{aligned}
H_{B} & =5.000+1.372-96.070-0.084 \\
& =-89.782 \mathrm{~m}
\end{aligned}
$$

Chúng tôi cũng đã tiến hành đo đạc thực nghiệm phương pháp chuyền độ cao qua giếng đứng xuống hầm bằng hai phương pháp truyền thống là phương pháp thước thép và phương pháp dây thép tại vị trí đo thực nghiệm máy TĐĐT. Kết quả độ cao điểm $\mathrm{B}$ đo được bằng phương pháp thước thép là $-89.780 \mathrm{~m}$ và kết quả đo được độ cao điểm $\mathrm{B}$ bằng phương pháp dây thép là -89.785m. (Xem bảng 2)

\section{Nhân xét:}

Từ kết quả so sánh ở bảng 2 cho thấy độ cao chuyền bằng máy TĐĐT so với độ cao chuyền bằng hai phương pháp truyền thống là phương pháp chuyền bằng dây thép và phương pháp chuyền bằng thước thép có sự sai lệch nằm trong giới hạn cho phép $(03 \mathrm{~mm})$ [1]. Kết quả thực nghiệm cho thấy có thể sử dụng máy TĐĐT để chuyền độ cao xuống hầm qua giếng đứng có chiều sâu lớn thay hai phương pháp truyền thống vẫn hay dùng là phương pháp chuyền bằng thước thép và phương pháp chuyền bằng dây thép, khắc phục được những khó khăn về mặt kỹ thuật thường gặp khi dùng hai phương pháp truyền thống trên.

\section{Kết luận}

Từ kết quả phân tích về lý thuyết và đo đạc thực nghiệm, chúng tôi rút ra một số kết luận sau đây:

- Hoàn toàn có thể sử dụng máy TĐĐT để chuyền độ cao qua giếng đứng từ mặt đất xuống hầm, đặc biệt hiệu quả khi công trình đường hầm có độ sâu lớn.

- Để kết quả chuyền độ cao xuống hầm được chính xác nhất, nên dùng dây dọi để xác định vị trí đặt gương phản xạ $O$ dưới hầm. Khi đo khoảng cách từ máy TĐĐT đến gương phẳng phụ $\mathrm{P}$ nên đo bằng chế độ đo không gương và đo khoảng cách từ máy TĐĐT đến gương phản xạ $\mathrm{O}$ nên đo bằng chế độ đo có gương. $O$

\section{Tài liệu tham khảo}

[1]. Phan Văn Hiến và nnk (2013), Giáo trình Trắc địa công trình ngầm. Trường Đại học Tài nguyên và Môi trường Hà Nội.

[2]. Trần Viết Tuấn và nnk (2016), Máy trắc địa và Đo đạc điện tử, Nxb Bách khoa Hà Nội.

[3]. Trần Viết Tuấn (2013), Nghiên cứu hoàn thiện phương pháp chuyền độ cao qua giếng đứng xuống hầm bằng máy Toàn đạc điện tử, Tạp chí KHKT Mỏ - Địa chất, số 44, 10-2013.

[4]. Trần Viết Tuấn (2013), Nghiên cứu ứng dụng máy Toàn đạc điện tử để chuyển độ cao qua giếng đứng xuống hầm khi thi công hầm đối hướng, Tạp chí Khoa học và Công nghệ Mỏ, số 2 - 2013. O

Bảng 2: So sánh kết quả chuyền độ cao theo ba phương pháp

\begin{tabular}{|c|c|c|c|c|c|}
\hline $\begin{array}{c}\text { Phương pháp } \\
\text { chuyền độ cao }\end{array}$ & $\begin{array}{c}\text { Chuyền độ cao } \\
\text { bằng thước } \\
\text { thép }\end{array}$ & $\begin{array}{c}\text { Chuyền độ cao } \\
\text { bằng máy } \\
\text { TĐĐT }\end{array}$ & $\begin{array}{c}\text { Chuyền độ } \\
\text { cao bằng dây } \\
\text { thép }\end{array}$ & $\begin{array}{c}\text { Chênh lệch giữa TĐĐT } \\
\text { và phương pháp bằng } \\
\text { thước thép }\end{array}$ & $\begin{array}{c}\text { Chênh lệch giữa } \\
\text { TĐĐT và phương } \\
\text { pháp bằng dây thép }\end{array}$ \\
\hline 1 & 2 & 3 & 4 & $5=3-2$ & $6=3-4$ \\
\hline Độ cao điểm B & $-89.780 \mathrm{~m}$ & $-89.782 \mathrm{~m}$ & $-89.785 \mathrm{~m}$ & $-02 \mathrm{~mm}$ & $+03 \mathrm{~mm}$ \\
\hline
\end{tabular}




\section{Summary}

Studying a solution for improving the working effectiveness of transferring altitudes via vertical shafts to tunnels when building big-depth tunnels

Diem Cong Huy

Vietnam Institute for Building Science and Technology (IBST) - Ministry of Construction (MOC)

This paper presents some studying results on the technical characteristics and solutions for transferring altitudes via vertical shafts to the tunnel, some experimental surveying results, from which the most suitable method for transferring the altitudes via the vertical shafts to the tunnel can be selected and applied when building the big-depth tunnels. $O$

\section{XÁC ĐịNH LƯỢNG BÓC THOÁT NƯỚC........}

[3]. Jackson, R. D., J. L. Hatfield, R. J. toán lượng bốc thoát hơi nước ở khu vực Reginato, S. B. Idso, and P. J. Pinter, Jr., phía bắc Việt Nam bằng sử dụng tư liệu Estimation of daily evapotranspiration from viễn thám", Kỷ yếu Hội thảo Khoa học Công one-time-of-day measurements. Agric. nghệ, Viện khoa học Đo đạc và Bản đồ, Hà Water. Manage. 7(3) (1983) 351-362. Nội, 2014.

[4]. Minh Le, Ke Luong Chinh, Tuong

[6]. ThS. Nguyễn Văn Hùng và các cộng Tran Ngoc, Hung Nguyen Van, Son Le Minh, Essessing the accuracy of land surface evapotranspiration estimated by Makkink's model based on solar radiation extracted from modis data, Proceedings of the 9th International Conference on Geoinformation for Disaster Management, Hanoi, Vietnam, 9-11 Dec. 2013.

[5]. Nguyễn Văn Hùng, Trần Ngọc Tưởng, Lương Chính Kế "Xác định hệ số tuyến tính của mô hình Makkink cho tính sự, 2016 "Mô hình ước tính bốc thoát hơi nước thực tế bằng tích hợp chỉ số thực vật NDVI của ảnh Modis với số liệu khí tượng cho địa hình miền Bắc Việt Nam", $K y ̉$ yếu Hội thảo khoa học Đo đạc và Bản đồ với ứng phó biến đổi khí hậu, Viện khoa học Đo đạc và Bản đồ, Hà Nội.

[7]. Roerink, G.J.; Su, Z.; Menenti, M. SSEBI: A simple remote sensing algorithm to estimate the surface energy balance. Phys. Chem. Earth B 2000, 25, 147-157. O 